\title{
Diagnóstico clínico: competencia axiológica clave en la formación profesional del estudiante de odontología
}

\section{Clinical diagnosis: key axiological competence in the professional training of the odontology student}

\author{
Magister Claudia Gabriela Otazú Aldana ${ }^{1}$ \\ claudia.otazu@epg.usil.pe \\ ORCID: https://orcid.org/0000-0001-9034-113X \\ Dra. Patricia Medina Zuta² \\ patricia.medina@epg.usil.pe \\ ORCID: https://orcid.org/0000-0002-6315-935 \\ Dr. C. Angel Deroncele Acosta ${ }^{3}$ \\ angel.deroncele@usil.pe \\ ORCID: https://orcid.org/0000-0002-0413-014
}

Fecha de recibo: 1/9/2019, fecha de aceptación:1/11/2019

\section{RESUMEN}

Introducción: La competencia de diagnóstico clínico, se revela como núcleo esencial de la práctica profesional del odontólogo, en tanto se constituye en premisa de una intervención clínica-odontológica eficiente, de ahí que desde el pregrado, el proceso formativo del estudiante de odontología deba garantizar el desarrollo de esta competencia desde sus potencialidades formativas y axiológicas, articulando un proceso de enseñanza- aprendizaje de conocimientos, habilidades, valores y valoraciones que le permitan al estudiante desplegar el diagnóstico clínico como un modo de actuación profesional, ético, responsable y sostenible.

Objetivo: El presente estudio despliega el método de análisis documental en la formulación de una heurística orientadora y una indagación estratégica, como soporte de una hermenéutica de sistematización de fuentes científicas vinculadas a la política educativa vigente en relación con la formación del estudiante de odontología.

Desarrollo: El estudio se perfila en la necesidad de fomentar una actitud de compromiso ético del estudiante de odontología como futuro agente de transformación social, ello supone la potenciación del pensamiento crítico-reflexivo, y el tener en cuenta la competencia de diagnóstico clínico desde una perspectiva axiológicoformativa.

\footnotetext{
${ }^{1}$ Universidad San Ignacio de Loyola, Perú.

${ }^{2}$ Universidad San Ignacio de Loyola, Perú.

${ }^{3}$ Universidad San Ignacio de Loyola, Perú.
} 
Conclusiones: El reto formativo de desarrollar la competencia de diagnóstico clínico requiere de una posición protagónica del estudiante en el proceso de enseñanzaaprendizaje, lo cual implica repensar el rol de facilitador y mediador del docente clínico, incorporando estrategias pedagógicas activas a su saber y hacer axiológico-formativo; constituyéndose esta comunicación reflexiva y valorativa entre estudiante y docente en el eje dinamizador de la competencia.

Palabras clave: diagnóstico clínico, competencia axiológica, pensamiento críticoreflexivo, formación profesional, estudiante de odontología.

\section{ABSTRACT}

Introduction: The competence of clinical diagnosis is revealed as an essential nucleus of the professional practice of the odontology, while it constitutes the premise of an efficient clinical-odontology intervention, hence, from undergraduate, the formation process of the odontology student must guarantee the development of this competence from its formative and axiological potentialities, articulating a teachinglearning process of knowledge, skills and values that allow the student to display clinical diagnosis as a professional, ethical, responsible and sustainable way of acting. Objective: The present study deploys the method of documentary analysis in the formulation of a guiding heuristic and a strategic inquiry, as support for a hermeneutic of systematization of scientific sources linked to the current educational policy in relation to the training of the odontology student.

Development: The study is outlined in the need to promote an attitude of ethical commitment of the odontology student as a future agent of social transformation, this implies the enhancement of critical-reflective thinking, and taking into account the competence of clinical diagnosis from a perspective axiological-formative.

Conclusions: The formative challenge of developing the clinical diagnostic competence requires a leading position of the student in the teaching-learning process, which implies rethinking the role of facilitator and mediator of the clinical teacher, incorporating active pedagogical strategies into his knowledge and practice. axiological-formative; constituting this reflective and evaluative communication between student and teacher in the dynamic axis of the competition.

Keywords: clinical diagnosis, axiological competence, critical-reflective thinking, professional training, odontology student

\section{Introducción}

En el siglo XXI todavía es latente el interés por el aprendizaje, el pensamiento críticoreflexivo y la resolución de problemas. La perspectiva cognoscitiva del aprendizaje se puede describir como una orientación filosófica con nociones básicas sobre la capacidad de aprender (Woolfolk, 2010).

98

Otazú, Medina, Deroncele, Diagnóstico clínico: competencia axiológica clave en la formación profesional del estudiante de odontología 
En la actualidad, en el proceso de enseñanza-aprendizaje, el docente universitario de carreras de salud necesita afrontar una serie de desafíos; los que van desde la propia práctica pedagógica hasta la enseñanza de los procedimientos clínicos especializados. La formación del estudiante de pregrado de odontología implica el desarrollo de competencias especializadas, fundamentalmente la competencia de diagnóstico clínico.

En este sentido, son reiterativas las ocasiones en las cuales los estudiantes han denotado ciertas dificultades para determinar planes de tratamiento pertinentes o una programación que sea acorde a los procedimientos clínicos prescritos para los pacientes. A pesar de tener los conocimientos adquiridos en cursos básicos de la carrera, muchas veces, los estudiantes no logran alcanzar la competencia de diagnóstico clínico. Incluso, en algunos casos, buscan ser prácticos e ir directamente al tratamiento del paciente, sin considerar las bases y sustentos que implican aspectos científicos y teóricos de alta relevancia.

Probablemente, la actual generación de estudiantes requiere alcanzar respuestas rápidas y prácticas antes que tratamientos que exijan mayor minuciosidad y complejidad. Lo que nos llevar a pensar, que puedan estar centrando sus prioridades en atribuir a la práctica profesional un sentido de productividad y beneficio económico, antes que de naturaleza ética más orientada al servicio y al bien común (Objetivos de Desarrollo Sostenible - ODS, 2000 - 2015).

Si examinamos este contexto en el marco de la perspectiva educativa actual, podemos mencionar el Informe a la Unesco de la Comisión Internacional sobre la educación para el siglo XXI, (Delors, Al Mufti, Carneiro, Chung, Geremek y Nazhao, 1996), titulado "La educación encierra un tesoro", en el cual se profundizan argumentos y explicaciones contundentes sobre los cuatro pilares de la educación.

Aprender a conocer: en relación a despertar la curiosidad intelectual del estudiante y estimular su sentido crítico; lo cual es fundamental en el diagnóstico clínico, ya que orienta la posibilidad de descifrar la realidad, y adquirir una autonomía de juicio, que los estudiantes de odontología necesitan involucrar en su bagaje profesional. Esto resulta en el aprender a aprender para ejercitar procesos cognitivos como la atención, la memoria y el pensamiento que desencadenen progresivamente otros procesos superiores de la mente.

Aprender a hacer: significa demostrar en forma operativa las competencias que se requieren en el nivel profesional, y poner en práctica un conjunto de acciones y procedimientos para la realización de diagnósticos eficientes.

Aprender a vivir juntos: a nivel de sociedad, los estudiantes necesitan lograr habilidades vinculadas a la interacción con sus pares, como la capacidad para afrontar y resolver conflictos de manera asertiva. Además, en los tiempos actuales, se demanda un potencial de interculturalidad como parte del bagaje que el ciudadano del mundo necesita proyectar en el campo personal profesional (OECD, 2018). 


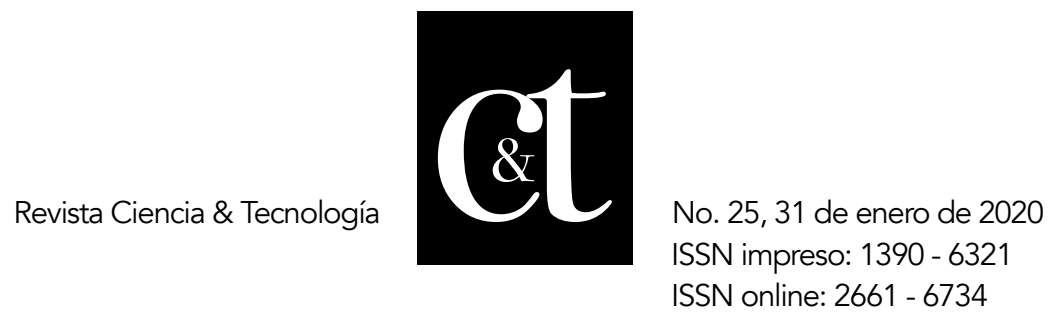

Aprender a ser: pilar de trascendencia, relacionado a que el estudiante desarrolle a plenitud su cuerpo y mente, su inteligencia, su sensibilidad, su sentido estético, su responsabilidad individual, y su espiritualidad; siempre considerando la capacidad de autonomía, de juicio y de responsabilidad personal. El estudiante necesita educarse y lograr un pensamiento autónomo y crítico para que pueda elaborar un juicio propio ante las situaciones que se presenten en la vida profesional (Chávez, 2017).

En medio de esta entramada de saberes que Delors (1996) plantea, está el estudiante universitario, el mismo que necesita ser una fuente de soluciones efectivas a los problemas clínicos, por lo que priorizar en su formación, el desarrollo del pensamiento crítico y reflexivo, es un acierto de sostenibilidad en sus potencialidades formativas y sobre todo en la generación de nuevos conocimientos y cambios orientados a la mejora y transformación social (Deroncele-Acosta, Medina-Zuta y Gross-Tur, 2020).

Según la Unesco (2016) en la declaración de Incheón, es imperioso que para el año 2030 se haya logrado una mayor cantidad de jóvenes y adultos con competencias técnicas y profesionales para acceder al trabajo decente. Sin embargo, a nivel de las carreras de formación del profesional en odontología del Perú, esto no estaría sucediendo; principalmente, debido a la proliferación de estas facultades y consecuentes ofertas de programas que tuvo lugar durante los años 90.

Esto nos convoca a replantearnos, ¿qué es lo que se viene buscando respecto a la formación inicial de los estudiante de odontología?, ¿de qué se trata la formación de estos profesionales? y ¿cómo puede garantizarse que efectúen diagnósticos eficientes?, esto último, si consideramos que es el punto medular del análisis que proponemos en este desarrollo hermenéutico.

Como futuros profesionales de odontología, los estudiantes de esta carrera, tendrían que acceder a un proceso formativo que sea garante de un posterior ejercicio profesional idóneo: atender y tratar personas, ejerciendo el pensamiento críticoreflexivo en el desarrollo de sus diagnósticos.

Esto les permitirá lograr un tratamiento exitoso y los hará protagonistas de un hacer competente que oriente permanentemente la transformación social, siempre en dependencia de las distintas configuraciones de la realidad y sus dificultades a nivel de la salud estomatológica. Por lo tanto, es imperioso analizar esta problemática educativa que busca asumir el reto formativo del diagnóstico clínico en la formación del estudiante de odontología.

En este sentido, el docente clínico, eje de este proceso, podría formularse ciertas interrogantes como punto de partida en su quehacer pedagógico: ¿cómo la formación básica puede influir en el proceso de enseñanza-aprendizaje y significar un reto para la educación superior de este profesional?, ¿es idóneo y suficiente el nivel de avance a nivel de pensamiento crítico y reflexivo que demuestra el estudiante como legado de la educación básica?, ¿qué es posible argumentar en relación a la competencia de diagnóstico clínico desde la mirada de los documentos rectores de la educación y formación que son parte de la política educativa actual? 


\section{Materiales y métodos}

El estudio realizado responde a la metodología del análisis documental, que tuvo como propósito principal la orientación de una hermenéutica problematizadora. De esta manera, se identifica el diagnóstico clínico como categoría central del análisis, sobre el cual se han dispuesto prácticas heurísticas y hermenéuticas (Londoño, Maldonado y Calderón, 2014; citado en Medina-Zuta y Deroncele-Acosta, 2019a); todas ellas vinculadas a la indagación y sistematización de fuentes de diferente naturaleza, con énfasis en documentos rectores de la política educativa glocal y global.

Se efectuó así, un proceso de construcción científico textual centrado en prácticas auto e inter-reflexivas (Medina-Zuta y Deroncele-Acosta, 2019b). Ello permitió develar la argumentación y realizar una síntesis panorámica de categorías vinculadas al proceso formativo del profesional estudiante de odontología, el rol fundamental del docente formador, entre otros elementos que permitieron un análisis profuso y una mayor claridad de lo planteado.

\section{Resultados y discusión}

EI conocimiento pedagógico: aspecto fundamental del proceso formativo especializado

El docente de la carrera de odontología en el Perú suele ser un profesional especializado en una determinada área clínica, como la cirugía, la endodoncia, la periodoncia, la implantación de prótesis, la ortodoncia, o la odontopediatría, entre las ramas más reconocidas. Se trata de un docente de odontología con experiencia clínica especializada, pero debido a limitaciones en su formación pedagógica, podría presentar dificultades en su forma de "llegar al estudiante"; generando con ello, posibles barreras en el proceso de enseñanza-aprendizaje.

En relación a ello, es importante destacar que para un docente formador, se hace propicio el conocimiento y comprensión de las teorías del aprendizaje, sobre todo las de perspectiva cognoscitiva y socio cultural. Según menciona Woolfolk (2010), los debates sobre la naturaleza del conocimiento, el valor de la razón y los contenidos de la mente se remontan a los antiguos filósofos griegos. Desde allí, tendríamos un despliegue de saberes integrados de naturaleza inter y multidisciplinar, que aportarían ampliamente al saber pedagógico que necesita incorporar y asegurar el docente clínico en su práctica habitual.

Desde finales del siglo XIX decayeron los estudios sobre la cognición y entró en auge el conductismo. Después de la segunda guerra mundial, se destacaron investigaciones vinculadas al desarrollo de habilidades humanas complejas, que generaron avances sobre la comprensión del desarrollo del lenguaje y estimularon nuevamente el resurgimiento de la investigación cognoscitiva. Los psicólogos educativos se interesaron en como las personas piensan, aprenden conceptos y resuelven problemas. En esta línea, Piaget, explica el cómo y por qué se construye el conocimiento, a través de ciertos procesos como la toma de consciencia, la abstracción y la autorregulación (Palomino, 2015). 
Asimismo, podemos mencionar a Vygotsky con su teoría socio histórico cultural, relacionada a las funciones psíquicas superiores, que ayudan a ilustrar la importancia de los procesos internos psicológicos en el desarrollo y en el propio aprendizaje (Palomino, 2015). Igualmente en los años 60, Ausubel generó el concepto del aprendizaje significativo, entendido en la integración de los nuevos conocimientos con los conocimientos previos del estudiante; determinándose un "anclaje" o un aprendizaje duradero, que ciertamente implica la complejidad en el avance del conocimiento como proceso continuo y cíclico.

En el camino de la psicología cognitiva, Bruner aduce que el estudiante construye conocimiento cuando realiza proposiciones, verifica hipótesis y realiza inferencias; todo ello a partir de su propio descubrimiento. Es por esto, que el aprendizaje es un proceso activo, de asociación, de construcción y de representación (Guilar, 2009; Medina-Zuta y Deroncele-Acosta, 2019a).

Al respecto, en la formación profesional en odontología, el proceso de enseñanzaaprendizaje puede fundamentarse en los mismos pilares. Estas consideraciones serían un foco de atención determinante en los niveles de conocimiento y manejo que necesita incorporar en su práctica el eje principal de este proceso formativo: el docente formador de los futuros estudiante de odontologías.

A pesar de ser un clínico con mucha experiencia, si el docente formador no logra que el estudiante se motive, reflexione, adquiera nuevos conocimientos el estudiante no alcanzará aprendizajes significativos, y por ende, no alcanzará la competencia de diagnóstico clínico. En la medida en que los estudiantes no desarrollen el pensamiento crítico-reflexivo, la problemática de desarrollar el diagnóstico clínico será latente.

\section{Las competencias profesionales del estudiante de odontología}

Si el proceso de enseñanza- aprendizaje fluye de manera efectiva, se lograrán todas las competencias profesionales necesarias para la atención de pacientes. A nivel internacional, es importante mencionar el Informe Final del Proyecto Tuning para América Latina (2004-2007), el cual dio a conocer las competencias profesionales de la salud. Sin embargo, en este documento observamos que son insuficientemente abordadas las competencias del profesional en odontología.

Se revelan limitaciones para una orientación oportuna de las competencias de estos estudiantes, y ello nos hace contrastar y analizar de manera muy concreta los aspectos de competencia del profesional de medicina con los del profesional de odontología.

En este sentido las competencias más importantes de un estudiante de medicina según este marco rector, estarían reconocidas en distintas capacidades como: efectuar diagnósticos sintomáticos, evaluar signos vitales, redactar la historia clínica e indicar y realizar tratamientos médicos. Dado ello, las capacidades descritas se pueden extrapolar a los estudiantes de odontología, que también realizan actividades similares con sus pacientes. 
A nivel de Perú, el Sistema Nacional de Evaluación, Acreditación y Certificación de la Calidad Educativa (Sineace, 2010), alude al colegio profesional de esta carrera como el referente del establecimiento de competencias que debe alcanzar un estudiante durante su proceso formativo. En esta misma orientación, el Colegio Odontológico del Perú (COP, 2010), también incluye dentro de sus normas, las competencias profesionales del cirujano dentista, explicitando como elemento prioritario la realización de diagnósticos que garanticen la salud estomatológica del paciente según los procedimientos normados y pre establecidos. Todo ello, sería la base de un plan de tratamiento estomatológico avalado en estos niveles de especialización.

Por consiguiente, es una prioridad que el estudiante de odontología se encuentre preparado para realizar diagnósticos e indicar alternativas de tratamiento que sean pertinentes a los distintos casos. En relación a la normativa presentada, el docente formador, necesitaría fomentar en el proceso de enseñanza-aprendizaje, que los estudiantes desarrollen una serie de habilidades y con ello, puedan alcanzar una práctica clínica eficiente, con énfasis en lo que corresponde a la competencia de diagnóstico clínico.

\section{La competencia de diagnóstico clínico}

Como bien se ha visto, el estudiante de odontología necesita ir afianzando sus competencias profesionales, y sabemos que entre ellas, el diagnóstico clínico cumple un papel fundamental. Pero, ¿qué entendemos por diagnóstico clínico en odontología? Sabemos que una competencia está conformada por diversas habilidades de orden superior, por lo tanto, la competencia puede ser definida como el conjunto de capacidades que apoyadas entre sí permiten ejecutar con éxito una determinada tarea académica, profesional o social en un escenario concreto (Sanz de Acedo, 2011).

Las competencias de toma de decisiones y de solución de problemas son algunas de las más desafiantes y complejas, es por esto que son conocidas como competencias superiores vinculadas al desarrollo del pensamiento. En los estudiantes de Educación Superior, estas competencias necesitan impulsarse y ejercitarse. En precisión de ello, el estudiante de odontología será competente en tanto pueda demostrar que puede solucionar un problema, lograr el diagnóstico de un paciente, y tomar decisiones sobre las diferentes estrategias a utilizar.

La competencia de saber tomar decisiones se puede definir como la capacidad para determinar algo, en función de entender su necesidad, analizando los factores que lo desencadenan, proponiendo alternativas, considerando sus consecuencias, es decir, discernir entre los pros y contras que puedan someter la elección de una mejor opción. En el mismo plano, la competencia orientada a saber solucionar problemas, se define como la capacidad para afrontar y resolver una situación conflictiva, analizando sus causas e ideando posibles salidas o soluciones a la misma, siempre verificando en ello el factor de eficacia. Entonces, resolver problemas atañe un proceso cognitivo más complejo que el de la toma de decisiones (Sanz de Acedo, 2011; Elangovan, GuzmanArmstrong, Marshall, \& Johnsen, 2018; Mamede et al., 2012). 


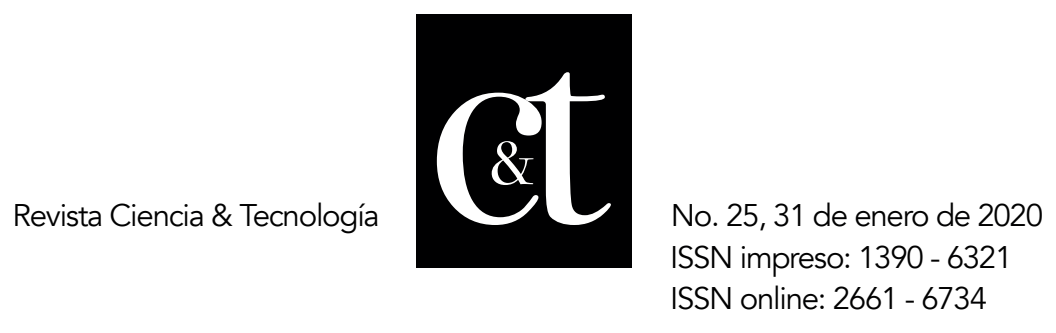

Ahora bien, si se examina la acepción del término diagnóstico en su definición más concreta, la Real Academia de la Lengua Española (RAE, 2020) la define como la determinación de la naturaleza de una enfermedad mediante la observación de sus síntomas. Pero, adscribiendo esta definición al campo de la odontología, podemos entender que el diagnóstico es saber determinar las enfermedades y patologías bucales mediante el análisis de síntomas y signos que presente el paciente (Newsome, Smales, \& Yip, 2012).

Frente a todo lo esbozado en esta parte del análisis, es significativo entender que la competencia de diagnóstico clínico integra los 4 saberes de Delors (1996): saber, hacer, ser y convivir enmarcando el rol de un futuro profesional que resuelva problemas de salud médica estomatológica proponiendo alternativas viables en los procesos de su propia evolución y empoderamiento profesional y en la transformación y sostenibilidad de una sociedad mundial cada vez más saludable. Integrando lo planeado anteriormente se asume como competencia profesional:

la configuración psicológica que integra lo afectivo-motivacional, lo actitudinal y lo cognitivo- metacognitivo, y se expresa en la movilización de los recursos laborales-profesionales en función de un desempeño profesional contextualizado eficiente, lo que explica su carácter relacional, socio-dinámico, situacional y actual" (Deroncele, 2015; p.74).

\section{El pensamiento crítico-reflexivo como aspecto central en la formación de competencias del estudiante de odontología}

Desde la perspectiva formativa en el contexto socio-educativo peruano, se asumen para este estudio los presupuestos de Deroncele, Nagamine y Medina (2020), relacionados con las "bases epistemológicas y metodológicas para el abordaje del pensamiento crítico en la educación peruana"; estos autores plantean que:

el desarrollo y potenciación del pensamiento crítico es un tema de trascendental importancia en la educación actual toda vez que ello garantiza una mejor activación de los procesos de aprendizaje, logrando sujetos más conscientes, críticos, creativos y autónomos, capaces de enfrentarse a situaciones laborales, académicas y de la propia vida cotidiana de manera eficiente" (Deroncele, Nagamine y Medina, 2020 p.70).

El estudiante de odontología necesita demostrar su capacidad crítica y reflexiva para el logro de la competencia de diagnóstico clínico. La importancia del pensamiento crítico-reflexivo va en estrecha relación con la necesidad de integrar competencias generales o básicas a través de las cuales el estudiante se disponga a efectuar una serie de procesos cognitivos vinculados a: recoger, analizar y evaluar críticamente la información que recoge (Otero, 2009; von Bergmann \& Shuler, 2019). Dentro de ello, sería oportuno explicar, que el estudiante de esta carrera necesita tener al respecto, una base previa, forjada desde la educación básica.

104

Otazú, Medina, Deroncele, Diagnóstico clínico: competencia axiológica clave en la formación profesional del estudiante de odontología 


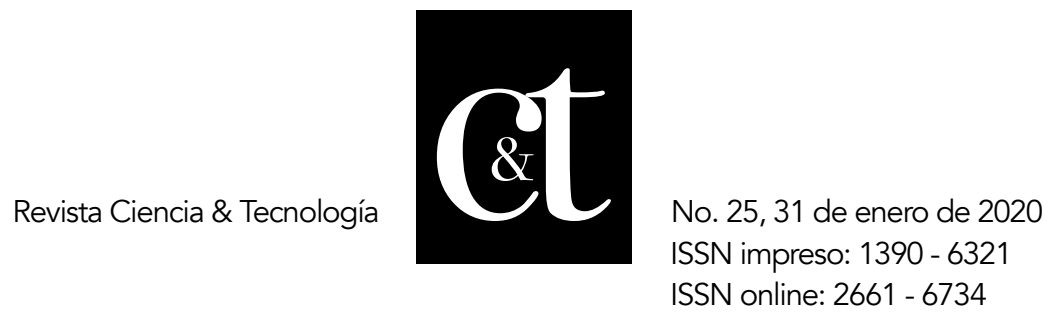

Pero, ¿qué tanto ello es consecuente en la línea de formación de la básica?, ¿los aspirantes a ser estudiante de odontologías acceden a una formación profesional inicial, habiendo consolidado bases cognitivas que denoten suficientes avances en su pensamiento crítico y reflexivo?

Ya hemos afirmado, que a nivel universitario, el pensamiento crítico reflexivo es un pilar decisivo en la práctica clínica del estudiante de odontología, sobre todo si se busca lograr diagnósticos eficientes. Pero si se tiene esta carencia, que se arrastra desde la etapa escolar, el diagnóstico clínico se vuelve un reto que supera en el estudiante la complejidad y la exigencia de procesos cognitivos superiores que necesita activar y consolidar. Todo ello representaría para el docente formador, un pasivo heredado desde la educación básica que necesita tener en cuenta en su tarea y en su rol pedagógico.

Al respecto, el Programme for International Student Assessment (PISA), constituye una confirmación constante de que las competencias básicas no se estarían logrando en etapas de escolaridad. Estudiantes de 15 años que fueron evaluados en varias oportunidades, estarían por debajo del promedio de esta prueba, cuando se tienen en cuenta habilidades de lectura, que incluyen interpretación, integración y evaluación de información de diferentes textos; siendo estas habilidades asociativas a situaciones de la vida diaria (Minedu, 2018). Por lo tanto, se presentan obstáculos en coadyuvar el pensamiento crítico como un elemento de soporte de la competencia de diagnóstico clínico. El graduado ideal planteado por Reimers (2015) en el foro de Dubai, distaría ampliamente en esta realidad contexto. Y es que la capacidad de reflexión crítica compromete disposición y estados reflexivos que la persona haya configurado en su habitualidad a lo largo del proceso formativo. Esto es, capacidad de auto-reflexión y de inter-reflexión (Medina-Zuta y Deroncele-Acosta, 2019b).

\section{La competencia de diagnóstico clínico en el Proyecto Educativo Nacional del Perú al 2021}

El Ministerio de Educación del Perú (Minedu) y el Consejo Nacional de Educación (CNE) han proyectado el Plan Nacional de Educación al 2021 (PEN). Documento en el cual hay una serie de objetivos estratégicos y resultados orientados a trazar una ruta para lograr la calidad educativa en el Perú. Si precisamos la política educativa 9, en la cual se destaca, la articulación entre la educación básica y la educación superior técnica o universitaria como condición de calidad educativa; podríamos entender el marco de análisis que tiene el proceso de formación del profesional de la salud, con especificidad en la carrera de odontología.

Si las instituciones educativas de formación básica, cumplieran los objetivos planteados por el PEN, seguramente los estudiantes de odontología tendrían una mayor disposición por ejemplo, a la capacidad crítica antes planteada y se continuaría con la secuencia de su desarrollo y aplicación en el ámbito de la carrera de odontología. Sin embargo, la articulación entre el nivel básico y superior no se hace evidente en esta intención. 


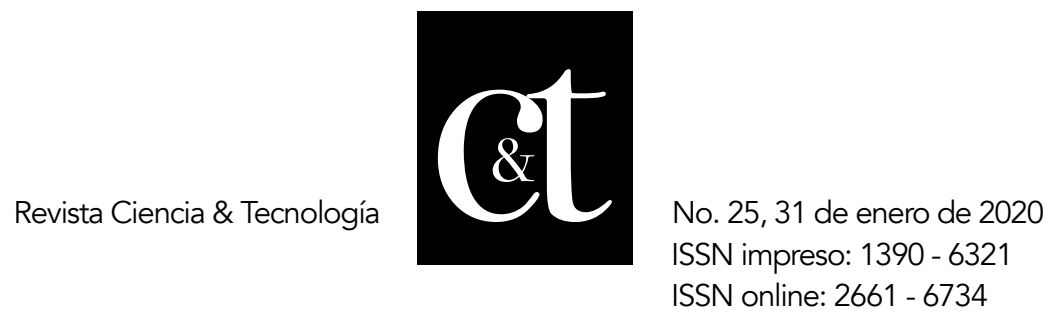

En esta misma línea del análisis, si especificamos el objetivo estratégico 2, referido a la pertinencia de estudiantes e instituciones que logren aprendizajes de calidad para afrontar las necesidades actuales, el profesional de odontología necesitaría hacer una lectura diagnóstica eficiente sobre la situación y necesidades de sus pacientes. En forma complementaria, el objetivo estratégico 5, menciona que la educación superior de calidad se convierte en factor favorable para el desarrollo y la competitividad nacional.

Esto proyectaría la gran necesidad de que el país cuente con profesionales que verdaderamente aporten al desarrollo y avance nacional. Se comprometen ideales en los cuales, los futuros profesionales de la odontología, tengan acceso a una educación superior de calidad, para llevar salud bucal a todas las zonas que se necesiten en el ámbito peruano.

Al respecto, el pilar de la investigación mencionado en la política 24, también es oportuno para la competencia de diagnóstico clínico, ya que el estudiante de esta carrera requiere implementar competencias investigativas, que le ayuden a realizar procesos de indagación y procesamiento constante de los nuevos conocimientos científicos y los que estén en avance. Indagar y conocer la información necesaria se convierte en un factor ineludible para elaborar un diagnóstico clínico.

La actualización constante que viabiliza la práctica investigativa se integra también con el manejo de las nuevas tecnologías; y es que la investigación y la tecnología van de la mano, si realmente queremos lograr un desempeño profesional competente en la práctica de todo profesional en estos tiempos. Por otro lado, asegurar un proceso de formación de calidad desde la intención de la política 25.1 planteada por el PEN, constituye para la educación superior universitaria y técnico profesional un reto que incluye al rol del docente formador. Aquí se hace relevante tomar en cuenta el desarrollo de metodologías de enseñanza que impulsen el pensamiento autónomo, crítico y creativo de los estudiantes y su capacidad para resolver problemas (Minedu y CNE, 2007).

En esta lógica, el docente que imparte cursos que son parte de la propuesta de formación del profesional en odontología, necesita ser foco de atención de las políticas educativas actuales. El desarrollo del pensamiento autónomo, crítico y creativo se convierte en un desafío constante para todo docente formador, quien debe garantizar que es capaz de orientar y concretar desde los cursos clínicos su consolidación e integración a una práctica profesional efectiva.

\section{Otros aspectos complementarios en la formación del estudiante de odontología}

Ya hemos visualizado que la capacidad crítica y reflexiva constituye una base de la competencia de diagnóstico clínico; sin embargo, hay que dilucidar otros aspectos fundamentales de este aprendizaje y entender su complejidad para orientar procesos pedagógicos de calidad. Al respecto, Hernández, Torres, Fang y Díaz-Caballero (2017), 
manifestaron que desde una visión educativa, identificar las características que requiere un graduado de odontología, ciertamente competente, compromete una práctica profesional en la cual se hayan consolidado las bases de una cultura de investigación constante, asi como capacidades de autorregulación y disposición a la participación frente a las demandas y tareas académicas.

Existe bibliografía que permite apoyar esta información, por ejemplo, Tweed, y Wilkinson, (2019) mencionan la relación que puede tener el conocimiento y la capacidad de raciocinio que tienen los estudiantes, sobre todo en los últimos años, en carreras médicas, que es comparable con un jurado, como sucede en un juicio, el jurado de manera grupal llega a un veredicto; así de forma colaborativa los estudiantes pueden llegar al diagnóstico final.

Asimismo, Shuler (2014), expone que los docentes formadores deben asegurar que sus estudiantes reciban una educación sólida, con una integración completa de conocimientos, habilidades y valores, para que los futuros profesionales estén bien preparados y sepan afrontar los cambios en la profesión que se producirán en los próximos cuarenta años.

\section{Conclusiones}

Después de analizar la problemática de la formación profesional del estudiante de odontología en Perú, se connota que asumir el diagnóstico clínico como una competencia axiológica clave en el proceso formativo desde el pregrado, tendrá un impacto positivo en el desempeño ético, responsable y sostenible del profesional de odontología, lo cual se expresa en un intervención clínica-odontológica eficiente.

Se asume como elemento dinamizador para el logro de este proceso formativo, la potenciación del pensamiento crítico-reflexivo como pilar esencial en la generación y consolidación de la competencia de diagnóstico clínico. En este sentido, la universidad debe promover un programa formativo garante de esta aspiración.

Se trata de lograr una formación profesional de agentes de cambio y transformación social que sean referentes y piezas clave en la comunidad donde se desempeñen profesionalmente. Por ello, es indispensable que la prioridad de formación de estos profesionales se centra en su carácter integral, en la responsabilidad social y en el factor ético, de ahí las potencialidades de la competencia de diagnóstico clínico desde una perspectiva axiológico-formativa.

Es decisivo, que el docente clínico especializado, se disponga a gestionar aspectos pedagógicos constitutivos de la formación por competencias, que le permitan constituirse como facilitador y mediador del proceso de enseñanza- aprendizaje de la competencia de diagnóstico clínico del estudiante de odontología, favoreciendo que éstos puedan lograr diagnósticos clínicos eficientes a partir del análisis y comprensión responsables de sujetos, situaciones y contextos específicos.

El reto en la formación profesional del estudiante de odontología, es comparable a un viaje, que necesita ser realizado en las más óptimas condiciones y asegurar que todos los pasajeros lleguen a su destino final a buen resguardo.

\section{7}

Revista científica Ciencia y Tecnología Vol 20 No 25 págs. 97-109

http://cienciaytecnologia.uteg.edu.ec 
El mejor diagnóstico clínico para la persona, es aquel que orienta una ruta clara y eficiente para resolver un problema de salud estomatológica. El profesional estudiante de odontología que infunde confianza es aquel que sabe proyectarse en su competencia de diagnóstico clínico: punto de partida para un desempeño de calidad.

\section{Referencias bibliográficas}

Chávez, N. (2017). Aprendizaje estratégico y metacognición. Educación: Revista de la Facultad de Ciencias de la Educación, 23, 91-99. https://doi.org/10.33539/educacion.2017.n23.1174

COP. (2010, octubre). Normas priorizadas de competencias profesionales del cirujano dentista. Recuperado de https://www.sineace.gob.pe/wpcontent/uploads/2018/08/Estudiante de odontología-NC-1.1.1-Identificar-lanecesidades-de-Taención.pdf

Delors, J., Al Mufti, I., Amagi, I., Carneiro, R., Chung, F., Geremek, B., \& Nazhao, Z. (1996). Informe a la Unesco de la comisión internacional sobre la educación para el siglo XXI: La educación encierra un tesoro. Madrid: Santillana, Ediciones UNESCO.

Deroncele Acosta, A., Medina Zuta, P., y Gross Tur, R. (2020). Gestión de potencialidades formativas en la persona: reflexión epistémica y pautas metodológicas. Universidad y Sociedad, 12(1), 97-104.

Deroncele Acosta, A., Nagamine Miyashiro, M., y Medina Coronado, D. (2020). Bases epistemológicas y metodológicas para el abordaje del pensamiento crítico en la educación peruana. Revista Inclusiones. 7(Número Especial), 68-87.

Deroncele-Acosta, A. (2015). Estrategia educativa para la formación profesional integral del psicólogo en el contexto organizacional. Tesis de Doctorado. Santiago de Cuba. Universidad de Oriente.

Elangovan, S., Guzman-Armstrong, S., Marshall, T. A., \& Johnsen, D. C. (2018). Clinical decision making in the era of evidence-based odontology. Journal of the American Dental Association (1939), 149(9), 745-747. https://doi.org/10.1016/j.adaj.2018.06.001

Guilar, M. (2009). Las ideas de Bruner: «de la revolución cognitiva» a la «revolución cultural». Educere, 13(44), 235-241. Recuperado de http://www.redalyc.org/articulo.oa?id $=35614571028$

Hernández, A., Torres, F., Fang, L., \& Díaz-Caballero, A. (2017). Estrategias de aprendizaje en estudiantes de odontología de una universidad pública en Cartagena, Colombia. Universitas Odontologica, 36(76), 20. https://doi.org/10.11144/Javeriana.uo36-76.eaeo

Mamede, S., van Gog, T., Moura, A. S., de Faria, R. M. D., Peixoto, J. M., Rikers, R. M. J. P., \& Schmidt, H. G. (2012). Reflection as a strategy to foster medical students' acquisition of diagnostic competence. Medical Education, 46(5), 464472. https://doi.org/10.1111/j.1365-2923.2012.04217.x

Medina Zuta, P., Deroncele Acosta, A. (2019a). La evaluación formativa desde el rol del docente reflexivo. Revista Maestro y Sociedad, 16(3), 597-610. 
Medina Zuta, P., Deroncele Acosta, A. (2019b). La construcción científico-textual en el posgrado: el desafío de la transdisciplinariedad y la reflexividad. Revista Maestro y Sociedad, 16(4), 829-838.

Ministerio de Educación de Perú, \& Consejo Nacional de Educación. (2007). Proyecto educativo Nacional al 2021. La educación que queremos para el Perú. Recuperado de http://www.minedu.gob.pe/DeInteres/xtras/PEN-2021.pdf

Ministerio de Educación de Perú. (2018). Marco de evaluación de la competencia lectora de PISA 2018. Recuperado de http://umc.minedu.gob.pe/wpcontent/uploads/2017/11/marco-fundamentacion-pisa.pdf

Newsome, P., Smales, R., \& Yip, K. (2012). Oral diagnosis and treatment planning: Part 1. Introduction. Br Dent $J, \quad 213, \quad$ 15-19. https://doi.org/doi:10.1038/sj.bdj.2012.559

OECD (2018). Marco de competencia global. Estudio PISA. Preparar a nuestros jóvenes para un mundo inclusivo y sostenible. PISA 2018. Gobierno de España. Ministerio de Educación, Cultura y Deporte.

Otero, E. (2009). El pensamiento crítico. Chile: Universidad Uniacc.

Palomino, C. (2015). Concepciones de los docentes acerca de la enseñanza de la metacognición en el aprendizaje en una I.E. de secundaria del distrito de El Agustino (Tesis de Maestría en Educación con mención en Currículo, Pontificia Universidad Católica del Perú). Recuperado de http://hdl.handle.net/20.500.12404/8492

Real Academia Española. (2020). Diccionario de la lengua española [en línea]. Disponible en: https://www.rae.es/

Sanz de Acedo, M. (2011). Competencias cognitivas en Educación Superior. MadridEspaña: Narcea.

Shuler, C. F. (2014). Dental school: Balancing education and training. Journal of Dental Education, 78(5), 655-656.

Sineace. (2010). Norma del competencia del cirujano dentista. Resolución 039-2010SINEACE/P.

Recuperado

de https://www.sineace.gob.pe/certificacion/certificacion-de-educacionsuperior/\#1534259012861-cd084bbe-d358

Tuning-América Latina. (2007). Reflexiones y perspectivas de la Educación Superior en América Latina. Informe final. Recuperado de http://tuning.unideusto.org/tuningal/index.php?option=com_weblinks\&Itemid =4

Tweed, M., \& Wilkinson, T. (2019). Student progress decision-making in programmatic assessment: Can we extrapolate from clinical decision-making and jury decisionmaking? BMC Medical Education, 19(1), 176. https://doi.org/10.1186/s12909019-1583-1

Unesco. (2016). Educación 2030. Declaración de Incheon y Marco de Acción para la realización del objetivo de Desarrollo Sostenible 4. Recuperado de https://unesdoc.unesco.org/ark:/48223/pf0000245656 spa

Von Bergmann, H., \& Shuler, C. F. (2019). The Culture of Certainty in Odontology and Its Impact on Dental Education and Practice. Journal of Dental Education, 83(6), 609-613. https://doi.org/10.21815/JDE.019.075

Woolfolk, A. (2010). Psicología educativa (11a.). México: Pearson Educación. 